\title{
Whole genome comparisons of Fragaria, Prunus and Malus reveal different modes of evolution between Rosaceous subfamilies
}

\author{
Sook Jung ${ }^{1 *}$, Alessandro Cestaro ${ }^{2}$, Michela Troggio ${ }^{2}$, Dorrie Main ${ }^{1}$, Ping Zheng ${ }^{1}$, Ilhyung Cho ${ }^{3}$, Kevin M Folta ${ }^{4}$, \\ Bryon Sosinski ${ }^{5}$, Albert Abbott ${ }^{6}$, Jean-Marc Celton ${ }^{7}$, Pere Arús ${ }^{8}$, Vladimir Shulaev ${ }^{9}$, Ignazio Verde $^{10}$, \\ Michele Morgante ${ }^{11}$, Daniel Rokhsar ${ }^{12}$, Riccardo Velasco ${ }^{2}$ and Daniel James Sargent ${ }^{2}$
}

\begin{abstract}
Background: Rosaceae include numerous economically important and morphologically diverse species. Comparative mapping between the member species in Rosaceae have indicated some level of synteny. Recently the whole genome of three crop species, peach, apple and strawberry, which belong to different genera of the Rosaceae family, have been sequenced, allowing in-depth comparison of these genomes.

Results: Our analysis using the whole genome sequences of peach, apple and strawberry identified 1399 orthologous regions between the three genomes, with a mean length of around $100 \mathrm{~kb}$. Each peach chromosome showed major orthology mostly to one strawberry chromosome, but to more than two apple chromosomes, suggesting that the apple genome went through more chromosomal fissions in addition to the whole genome duplication after the divergence of the three genera. However, the distribution of contiguous ancestral regions, identified using the multiple genome rearrangements and ancestors (MGRA) algorithm, suggested that the Fragaria genome went through a greater number of small scale rearrangements compared to the other genomes since they diverged from a common ancestor. Using the contiguous ancestral regions, we reconstructed a hypothetical ancestral genome for the Rosaceae 7 composed of nine chromosomes and propose the evolutionary steps from the ancestral genome to the extant Fragaria, Prunus and Malus genomes.

Conclusion: Our analysis shows that different modes of evolution may have played major roles in different subfamilies of Rosaceae. The hypothetical ancestral genome of Rosaceae and the evolutionary steps that lead to three different lineages of Rosaceae will facilitate our understanding of plant genome evolution as well as have a practical impact on knowledge transfer among member species of Rosaceae.
\end{abstract}

Keywords: Rosaceae, Comparative genomics, Evolution

\section{Background}

The Rosaceae is one of the most economically important and morphologically diverse plant families with over 90 genera containing more than 3000 species. The family contains three sub-families; the Dryadoideae, the Rosoideae and the Spireaeoideae, with the economicallyimportant genera Prunus and Malus contained within the Spireaeoideae, whilst Fragaria is a member of the

\footnotetext{
* Correspondence: sook_jung@wsu.edu

'Department of Horticulture and Landscape Architecture, Washington State University, Pullman, WA 99164, USA

Full list of author information is available at the end of the article
}

Rosoideae [1]. The base chromosome number of the many genera within the family ranges from $x=7$ to $x=$ 17 , and recent research has suggested that the ancestral chromosome number for Rosaceae may have been $x=9$ $[2,3]$. As in many other plant families, comparative genomics will enhance our understanding of genome structure and function and the evolutionary forces that have led to the current chromosomal configurations of the numerous Rosaceous species, and in turn to the mechanisms responsible for the wealth of morphological diversity encompassed by the family. An understanding of the degree of conservation of genome structure and function
C Biomed Central

(c) 2012 Jung et al; licensee BioMed Central Ltd. This is an Open Access article distributed under the terms of the Creative Commons Attribution License (http://creativecommons.org/licenses/by/2.0), which permits unrestricted use, distribution, and reproduction in any medium, provided the original work is properly cited. 
between related genera will enable inferences to be made about the genomic positions of genes controlling common traits among genera and permit information gained in one species to inform investigations in another.

The recent availability of whole genome sequences has permitted the delineation of syntenic blocks at high resolution and from this the evolutionary history in plant lineages can be inferred. In the grasses, paleogenomic modeling, using sequences of the maize, rice, and sorghum genomes as well as large sets of genetically mapped genes in wheat and barley, led to the proposal of an ancestral grass karyotype for the five ancestral chromosomes $[4,5]$ from which all modern grass genomes evolved. The recent sequencing of the Brachypodium genome [6] revealed a whole-genome paleo-duplication in Brachypodium chromosomes, whilst comparisons of the Brachypodium, rice and sorghum genome sequences revealed orthologous relationships that were consistent with the evolution of the extant Brachypodium genome from an ancestral genome containing five chromosomes.

Similarly, in the dicots, whole genome sequencing has revealed patterns of genome evolution that it had not been possible to detect using comparative mapping of orthologous markers. The sequencing of the grapevine genome [7] and its comparison to the genomes of Arabidopsis and poplar permitted the identification of a paleo-hexaploidisation event in the common lineage of the three species which occurred after the monocotyledonous and dicotyledenous plant lineages diverged. This hexaploidisation event had not previously been identified, despite the whole genome sequences of Arabidopsis and poplar being available for some time $[8,9]$. This was primarily due to the subsequent polyploidisation events that had occurred in the genomes of these species (once in the case of poplar, and twice in the case of Arabidopsis) since they diverged from a common ancestor. Thus, analyses based on higher levels of resolution, particularly those based on whole genome sequence data, reveal evermore complex patterns of genome evolution between species, but at the same time provide compelling evidence to support models of genome evolution and deduced ancestral chromosomal configurations.

So far no studies have been performed that have compared whole genome sequences of plant species that belong to different genera of the same family. In Rosaceae, as well as in other economically important plant families including Poaceae, Solanaceae, Brassicaceae and Fabaceae [10-14], the comparative genomics studies have been performed using conserved genetic markers. Dirlewanger et al [15] first identified high levels of conservation of marker presence and order between three of the eight linkage groups of the Prunus reference map [16], and seven of the 17 linkage groups of the apple map [17], demonstrating that markers mapping to a single Prunus linkage group were located on two homeologous linkage groups on the Malus linkage map and that large conserved syntenic blocks were clearly identifiable within the two genera. A number of other studies were also performed using PCRbased markers that had been developed from both Malus and Fragaria, which were applied to comparative mapping between Prunus and these other members of the Rosaceae $[18,19]$. High level of co-linearity within the sub-family Maloideae between the genomes of Malus and Pyrus has also shown by comparative mapping using simple sequence repeat (SSR) markers [20]. Vilanova et al [2] reported a genome-wide inter-generic comparison of genetically mapped orthologous markers between diploid Fragaria and Prunus showing sufficiently well conserved macro-synteny to enable the reconstruction of a hypothetical ancestral genome for Rosaceae containing nine chromosomes. The study however also revealed a number of large-scale chromosomal rearrangements, including translocations of large syntenic blocks and numerous fusionfission events that had occurred in the evolutionary history of the two genera. More recently, using the whole genome sequence from the apple cultivar 'Golden Delicious' [21] and sequence data from 1,473 markers mapped in Prunus and Fragaria, including Rosaceous conserved orthologous sequences (RosCOS) [22], Illa et al [3] performed a genome-wide comparison between all three genera. Analyses based on the positions of the 129 markers revealed clear, conserved, syntenic blocks that were common to all three genomes, with a single syntenic block in Prunus corresponding to one or two syntenic regions in Fragaria, and two or four syntenic regions in apple. Illa et al [3] reconstructed a hypothetical ancestral genome for the Rosaceae containing nine chromosomes $(x=9)$, consistent with the report of Vilanova et al [2]. The data suggested that the resolution of studies based on modest numbers of markers was perhaps not sufficient to elucidate the true number of small scale genomic inversions that have taken place in genome evolution within the Rosaceae, which may have played an important role in speciation within the family. Thus, an evaluation of the conservation of synteny between Fragaria, Malus and Prunus based on whole genome sequence data may reveal much about sequence evolution in this closely-related, yet morphologically diverse family that has been hitherto undetected.

The genomes of three Rosaceous genera of significant economic importance, Fragaria [23], Malus [21] and Prunus [24] have recently been sequenced, presenting an exciting opportunity for high-resolution genome comparison. Here we report results from comparison of whole genome sequences of the three species of Rosaceae and the genome of Vitis vinifera, included as an outgroup species representing a basal rosid genome. We were able to identify the orthologous regions among the three Rosaceous species at a much higher-resolution than has 
previously been reported. This higher-resolution enabled us to detect different patterns of genome evolution between the sub-families of Rosaceae. Furthermore, we reconstructed a hypothetical Rosaceae ancestral genome using the Multiple Genome Rearrangements and Ancestors (MGRA) algorithm and further manual analyses.

\section{Results and Discussion}

\section{Evaluation of orthologous regions between taxon pairs}

The RosCOS markers used previously by [3] are a useful resource in comparative genome alignment and as such revealed insights into the patterns of genome evolution on a macro-syntenic scale in that study. Since the RosCOS are an important resource for future comparative studies, we anchored them to the orthologous regions (ORs) identified in this investigation (Additional file 3: Table S1). However, since orthologous genes in two species do not necessarily reside in large orthologous regions of the genome, using a relatively small set of orthologous sequences (as in the case of the RosCOS markers) in the detection of microsynteny would only be possible in genomic regions where the order of a large number of orthologs is conserved among related genomes. With only 800 mapped RosCOS available for study, it was difficult to detect orthologous regions at very high levels of resolution. Capitalising on the availability of whole genome sequences with many more predicted genes $(27,243$ in peach, 33,264 in strawberry and 43,335 in the primary assembly of apple), along with Mercator [24], which selects one to one orthologous regions based on the large numbers of exons available for study, meant that we were able to detect the conservation of synteny between the genomes at a much finer level in this investigation than in previous studies.

Thus, the evolutionary history of Rosaceous genomes was investigated through the detection of ORs between Prunus and Fragaria or Malus, using Mercator [25]. A total of 1281 ORs were obtained in the comparison between Prunus and Fragaria, with the longest region of 1.7 Mb of PC3 and 1.4 Mb of FC6 (Table 1). The mean number of matching exons in each OR was 17 and the mean lengths of ORs were $98.8 \mathrm{~kb}$ in Prunus and $98.4 \mathrm{~kb}$ in Fragaria (Table 1). Figure 1 shows the ORs between Prunus and Fragaria (A) and Prunus and Malus (B). In most cases, each peach chromosome showed major orthology to one strawberry chromosome, but to two or more apple chromosomes, clearly indicating that the whole genome duplication (WGD) in apple occurred following the divergence of the three genera. The ortholgous relationships between chromosomes of Fragaria and Prunus were clear, with the majority of ORs on Prunus chromosomes PC2, PC3, PC4, PC5, and PC8 each corresponding to single homologous chromosome in Fragaria, FC7, FC6, FC3, FC5, and FC2, respectively. The majority of ORs on PC7 corresponded to two Fragaria chromosomes, FC1 and FC6, and those on PC6 corresponded to three regions of the Fragaria genome on FC1, FC3 and FC6. The Prunus ORs on PC1 were the most widely distributed within the Fragaria genome, with ORs corresponding to multiple homologous chromosomal regions, but with one major syntenic relationship with FC4 (Figure 1A, Table 2).

The analysis between Prunus and Malus produced fewer, but larger ORs with a greater number of matching exons. The smaller number of ORs may reflect the fact that the primary assembly of apple does not include all the predicted genes sequenced. A total of 349 ORs were obtained, with the longest region of $6.6 \mathrm{Mb}$ of PC3 and $7.5 \mathrm{Mb}$ of MC9 (Table 1). The mean number of matching exons in ORs was 23 and the mean lengths of ORs were $200.9 \mathrm{~kb}$ in Prunus and $260.5 \mathrm{~kb}$ in Malus (Table 1). At the chromosome level, the analysis revealed more complex relationships between the two genera than between Prunus and Fragaria. ORs on PC3 and PC5 each corresponded to ORs on two major Malus chromosomes, MC9 and MC17, and MC6 and MC14, respectively. The two sets of Malus chromosomes, MC9/MC17 and MC6/ MC14, were two of the chromosome doublets that contain large syntenic regions indicative of the recent WGD in Malus lineage which agrees with previous hypotheses that the Malus genome went through relatively recent Pyreae-specific WGD [3,21], that occurred following the divergence of the Malus and Prunus lineages, as no evidence of such a WGD is present in the strawberry and peach genomes $[23,24]$. Orthologous regions in PC2 corresponded to major ORs on three Malus chromosomes, MC1, MC2 and MC7. ORs on PC1, PC4, and PC7 each corresponded to ORs on four Malus chromosomes, whilst ORs on PC6 corresponded to ORs on multiple Malus chromosomes (Figure 1B, Table 2). The observation that each chromosome of Prunus corresponded to ORs in two or more chromosomes of Malus, even though Mercator detects ORs in one to one relationships, suggests both sets of chromosomes generated by WGD retained orthologous relationships to their corresponding Prunus chromosomes. It also suggests that both of the two sub-genomic regions generated by WGD have retained a similar level of conservation of orthology. When the Malus chromosomes were divided into subgenome 1 and 2 prior to the analyses (see Materials and Methods) so that Mercator could find ORs in each Malus subgenome, 706 ORs were detected (Table 1). The whole genome duplication of Malus alone however does not account for the higher number of rearrangements that occurred since Prunus and Malus diverged from a common ancestor. Since the ancestor of the genus Fragaria diverged from a common ancestor shared by both Malus and Prunus, it is more likely that there have been more instances of large-scale chromosomal fission in the 
Table 1 Number and length of orthologous regions (ORs) in two-genome and three genome comparisons

\begin{tabular}{lllll}
\hline Orthology Analysis & $\begin{array}{l}\text { No. } \\
\text { OR }\end{array}$ & $\begin{array}{l}\text { Mean No. Matching } \\
\text { Exons }\end{array}$ & $\begin{array}{l}\text { Mean Length in Kb (Prunus| } \\
\text { Fragaria|Malus) }\end{array}$ & $\begin{array}{l}\text { Largest Length in Mb (Prunus| } \\
\text { Fragaria|Malus) }\end{array}$ \\
\hline Prunus and Fragaria & 1281 & 17 & $98.8|98.4| \mathrm{NA}$ & $1.7|1.4| \mathrm{NA}$ \\
\hline Prunus and Malus & 349 & 23 & $200.9|\mathrm{NA}| 260.5$ & $6.1|\mathrm{NA}| 7.5$ \\
\hline $\begin{array}{l}\text { *Prunus and Malus (Split into two } \\
\text { sub_genomes) }\end{array}$ & 706 & 22 & $175.9|\mathrm{NA}| 222.9$ & $5.5|\mathrm{NA}| 9.1$ \\
\hline Prunus, Fragaria and Malus & 1399 & ${ }^{* * 27}$ & $149.4|133.5| 82.4$ & $3.5|1.3| 2.6$ \\
\hline
\end{tabular}

*The Malus chromosomes were divided into sub-genome 1 and 2 prior to the analyses (see Materials and Methods) so that Mercator would find ORs in each Malus subgenome.

**Number includes the matching exons in two of the three genomes compared.

Malus lineage than the occurrence of multiple, yet independent fusion events in the Prunus and Fragaria lineages to derive the extant genome structure that is evident in the three genera today. More instances of largescale chromosomal fission may be a consequence of, or related to, the WGD that occurred in Malus lineage. Some of the rearrangements, however, may have resulted from the potential errors during genome sequencing and assembly.

\section{Evaluation of orthologous regions between Fragaria, Malus and Prunus}

The evolutionary relationships among the three Rosaceous species studied were analysed further by investigating ORs shared amongst all three genera in addition to those detected in each taxon pair. In total 1399 regions that were orthologous in all three genera were identified. The list of ORs with their positions and orientations in each genome are given in Table S1. Table S2 lists the size of ORs and the number of exons in each genome. The ORs contained 667 out of 855 RosCOS that have been anchored to the peach genome and 616 of the total 1399
ORs contained anchored RosCOS markers. The list of RosCOS markers, their anchored positions and their matching ORs are provided in Table S3. The longest OR in Prunus and Fragaria was OR 627 spanning $3.5 \mathrm{Mb}$ in PC8 and $1.3 \mathrm{Mb}$ in FC2 with an OR in MC9. The longest OR in Malus was $2.6 \mathrm{Mb}$ in MC4 with ORs in PC6 and FC6 (Table 1). OR 627 contained 1318 exons and 316 genes in Prunus, 998 exons and 200 genes in Fragaria, and 92 exons and 21 genes in Malus, respectively. The numbers of sequences in OR 627 with matches in other genomes were 125 exons and 62 genes in Prunus, 121 exons and 57 genes in Fragaria, and 21 exons and 6 genes in Malus, respectively. Table S4 lists all the genes and exons in OR 627 in each genome with their positions. The longest ORs in each genome and size distributions of the ORs are given in Table S5.

When multiple species are used, as in this analysis, pairwise homology maps can be utilized to build orthology maps for multiple species, as Mercator will find orthologous segments even if some anchors are missing in one of the species. The analysis thus resulted in the detection of additional orthologous regions that were
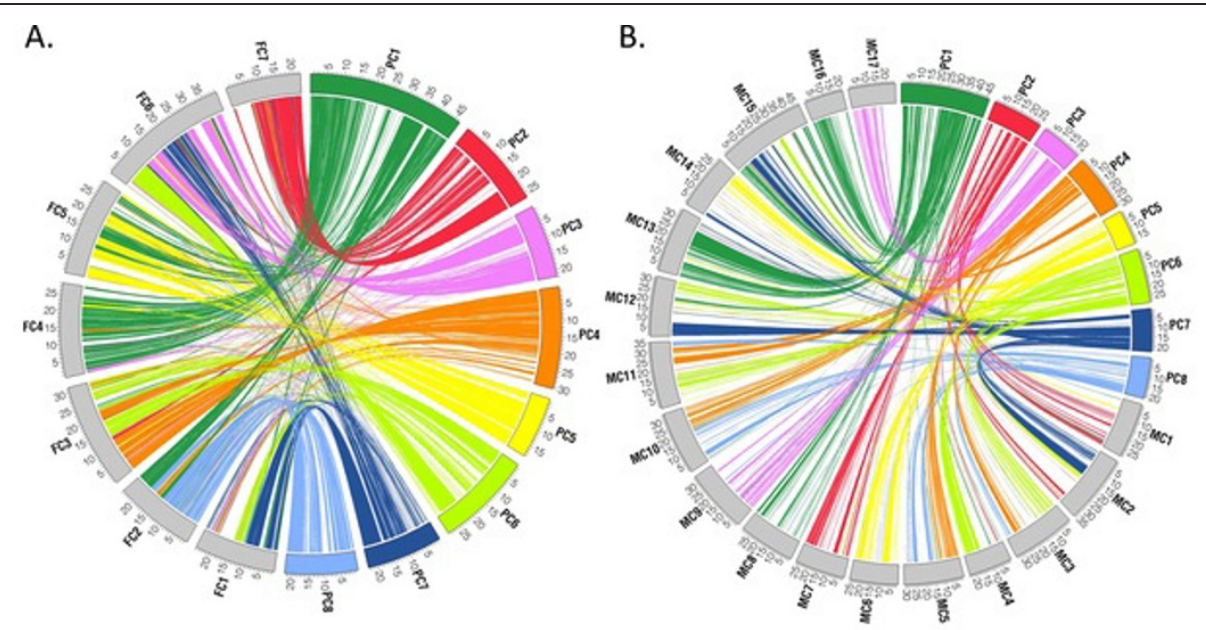

Figure 1 Orthology map identified between three Rosaceous genera based on whole genome sequence analysis. The lines link one to one orthologous regions, identified using Mercator program [25]. A. Comparison between Prunus and Fragaria, B. Comparison between Prunus and Malus. Data were plotted using Circos [42]. Colors for plots A and B follow the same pattern based on Prunus chromosomes. 
Table 2 Major orthologous chromosomes among Prunus, Fragaria and Malus

\begin{tabular}{lll}
\hline Prunus & Fragaria & Malus \\
\hline PC1 & FC2, FC4, FC5 & MC13/MC16, MC8/MC15 \\
\hline PC2 & FC7 & (MC1, MC2)/MC7 \\
\hline PC3 & FC6 & MC9/MC17 \\
\hline PC4 & FC3 & MC3/MC11, MC5/MC10 \\
\hline PC5 & FC5 & MC14/MC6 \\
\hline PC6 & FC1, FC3, FC6 & MC2/MC15, MC3/MC11, MC4/MC12 \\
\hline PC7 & FC1, FC6 & MC2/MC15, M14/M12 \\
\hline PC8 & FC2 & MC5/MC10, MC3/MC11 \\
\hline
\end{tabular}

The orthologous chromosomes were identified based on the result from orthology analysis using whole genome sequences (Figure 1).

not detected when the taxon pairs were investigated separately (Table 1 ). The comparison of ORs from the two-species analyses and the comparison of ORs from the three-species analysis are shown in Figure 2. Figure 2A shows ORs between PC2 and chromosomes of Fragaria and Malus, detected by separate taxon pair analyses. Figure 2B shows the same ORs shown in Figure 2A as well as the ORs shared between all three species. Blue lines link the ORs shared by all three species, red lines link ORs between Prunus and Fragaria only, and green lines link ORs between Prunus and Malus only. The figures showing ORs in the other seven Prunus chromosomes are shown in Additional file 1: Figure S1. The presence of red lines and green lines in Figure 2B shows that some ORs remain syntenic only between two species, as expected. The comparison of Figure 2A, B also shows additional ORs, which were not detected by the analyses of single taxon pairs. Most notable were the large numbers of additional ORs between Prunus and Malus that were detected in the three-species analysis. The additional ORs that were detected mostly resided in chromosomes that did not display major orthologous relationships with chromosome PC2 (Figure 2B, Table 2). This result suggests that content and/or order of the genes in ORs that reside on non-orthologous chromosomes went through more rearrangements than those in highly orthologous regions, masking their ancestral origins.

\section{Comparison of orthologous regions in major orthologous and non-orthologous chromosomes}

Further characterization and comparison of ORs in orthologous and non-orthologous chromosomes was performed through an examination of the size and the syntenic quality of the ORs that were conserved in all three species. Syntenic quality was defined as twice the number of matching exons divided by the total number of exons in both segments. The percentage identity (PID) and the bit score of the BLAT matches were also compared. Table 3 shows that the syntenic quality is higher in ORs between major orthologous chromosomes of Prunus and Malus (21.8\%) than those between non-orthologous chromosomes (16.8\%). The ORs from both groups however, had similar PIDs and bit scores between BLAT matches. We did not observe many differences in syntenic quality, PID and bit scores between major orthologous and non-orthologous regions in the analysis between the Prunus and Fragaria genomes, suggesting that chromosomal regions transposed by interchromosomal rearrangements in Malus have gone through more changes in terms of gene content and/or gene order, but not in terms of gene sequences. A WGD
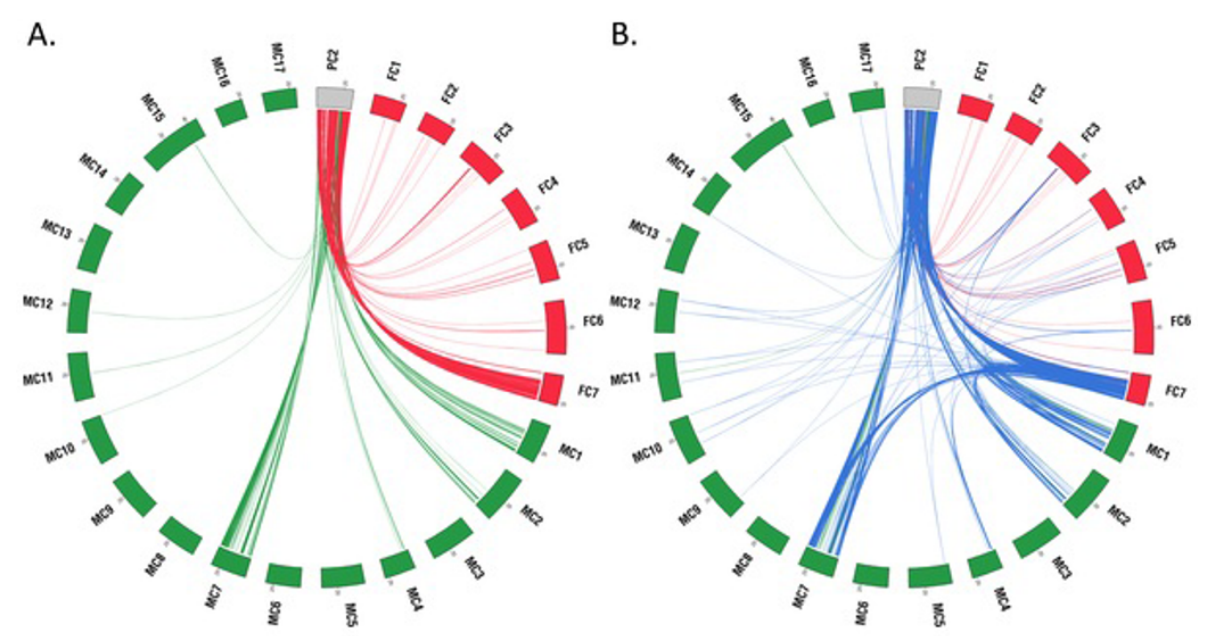

Figure 2 Comparison of orthologous regions (OR) from two-species analyses and those from the three-species analysis. A. ORs between PC2 and chromosomes of Fragaria and Malus, detected from two separate analyses. B. The same ORs shown in A as well as ORs that are shared by all three species. Blue lines link the ORs shared by all three species, red lines link ORs between Prunus and Fragaria only, and green lines link ORs between Prunus and Malus only. Data were plotted using Circos [42]. 
Table 3 Comparisons of orthologous regions (ORs) in major orthologous chromosomes with those in non-orthologous chromosomes

\begin{tabular}{|c|c|c|c|c|c|c|c|}
\hline ORs in & $\begin{array}{l}\text { No. } \\
\text { OR }\end{array}$ & $\begin{array}{l}\text { Mean length in kb } \\
\text { (Prunus| Fragaria) }\end{array}$ & $\begin{array}{l}\text { Mean No. Exons } \\
\text { (Prunus| Fragaria) }\end{array}$ & $\begin{array}{l}\text { Mean No. } \\
\text { Matching } \\
\text { Exons }\end{array}$ & $\begin{array}{l}\text { Mean Syntenic } \\
\text { Quality (\%) }\end{array}$ & $\begin{array}{l}\text { Mean } \\
\text { PID (\%) }\end{array}$ & $\begin{array}{l}\text { Mean Bit } \\
\text { Score }\end{array}$ \\
\hline $\begin{array}{l}\text { Orthologous chromosomes } \\
\text { between Prunus and Fragaria }\end{array}$ & 1261 & $151.0 \mid 137.3$ & $110 \mid 386$ & 27 & 23.6 & 87.1 & 137.3 \\
\hline non-orthologous chromosomes & 138 & 134.7|99.1 & $90 \mid 86$ & 23 & 24.3 & 87.5 & 134.3 \\
\hline All chromosome & 1399 & $149.4 \mid 133.5$ & $108 \mid 356$ & 27 & 23.6 & 87.1 & 137.1 \\
\hline ORs in & $\begin{array}{l}\text { No. } \\
\text { OR }\end{array}$ & $\begin{array}{l}\text { Mean length in kb } \\
\text { (Prunus } \mid \text { Malus) }\end{array}$ & $\begin{array}{l}\text { Mean No. Exons } \\
\text { (Prunus| Malus) }\end{array}$ & $\begin{array}{l}\text { Mean No. } \\
\text { Matching } \\
\text { Exons }\end{array}$ & $\begin{array}{l}\text { Mean Syntenic } \\
\text { Quality (\%) }\end{array}$ & $\begin{array}{l}\text { Mean } \\
\text { PID (\%) }\end{array}$ & $\begin{array}{l}\text { Mean Bit } \\
\text { Score }\end{array}$ \\
\hline $\begin{array}{l}\text { Orthologous chromosomes } \\
\text { between Prunus and Malus }\end{array}$ & 1181 & $133.4 \mid 87.6$ & $103 \mid 52$ & 26 & 21.8 & 89.6 & 143.3 \\
\hline non-orthologous chromosomes & 218 & $236.0 \mid 54.6$ & $136 \mid 35$ & 29 & 16.8 & 90.0 & 139.7 \\
\hline All chromosome & 1399 & $149.4 \mid 82.4$ & $108 \mid 49$ & 27 & 21.0 & 89.7 & 142.8 \\
\hline
\end{tabular}

Major orthologous chromosomes between Prunus and Fragaria/Malus are listed in Table 2. Regions that are conserved in all three genomes are considered in this comparison

event followed by massive gene loss, neofunctionalization of genes and other chromosomal changes have been observed in the evolutionary history of extant lineages, including yeast, plant and vertebrates [26-31]. The differences observed may be a consequence of the fact that the Malus genome has gone through a recent WGD and as a result has the highest number of predicted genes of any genome sequenced to date [21]. Thus Malus may have a greater degree of flexibility in the level of change in gene content and/or gene order that its genome can permit due to two copies of each gene being present than could be tolerated within the Fragaria genome., The syntenic quality between the two taxon pairs, however, was similar: $23.6 \%$ and 21.1\% for Prunus/Fragaria and Prunus/Malus, respectively (Table 3 ).

\section{Detection of conserved ancestral regions}

Reconstruction of a hypothetical ancestral genome for Rosaceae was performed using the MGRA (Multiple Genome Rearrangements and Ancestors) algorithm [32]. The Prunus and Fragaria genomes were used in the analysis with the Vitis genome as an outgroup. The Malus genome was not included in the MGRA analysis due to the fact that the primary assembly of apple did not include all the predicted genes sequenced. MGRA did not predict the number of chromosomes the ancestral genome contained, but it identified 49 CARs (Contiguous Ancestral Regions) that existed before the divergence of the Prunus, Fragaria and Malus genomes from a common ancestor. Each CAR represents a chromosomal region of the genome of the common ancestor of Prunus and Fragaria. The ancestral origins of the extant Malus chromosomes were inferred through a comparison of corresponding ORs in the Malus and Prunus genomes. Figure 3 shows the chromosomes of Prunus, Fragaria, and Malus, in which the 49 CARs are depicted in different colors. The results show that chromosomes of Fragaria are composed of many small chromosomal regions that originated from different ancestral CARs compared to those of Malus and Prunus (Figure 3), suggesting that the Fragaria genome went through a greater number of small scale rearrangements compared to the genomes of the other genera since they diverged from a common ancestor (Figure 3). Table 4 shows that the number of breaks between the chromosomal regions originating from different CARs in Fragaria is over two times greater than that in Malus and over 1.5 times greater than that in Prunus. The genomes of the diploid and the octoploid Fragaria that have been investigated to date through comparative mapping have been shown to be largely collinear $[33,34]$, however, whether the occurrence of small chromosomal rearrangements is common in the entire Fragaria lineage or restricted to species closely related to $F$. vesca would require further investigation.

\section{Reconstruction of hypothetical Rosaceae ancestral genome}

Since the genus Fragaria split from the common ancestor of Malus and Prunus before those species diverged, if regions with the same ancestral origin reside in the same chromosome of both Prunus and Fragaria, but in different chromosomes of Malus, we can infer that the those chromosomes of Malus were generated by a fission event. Likewise, if regions with the same ancestral origin reside in the same chromosome of Prunus but in different chromosomes of Malus and Fragaria, we can infer the chromosome of Prunus was generated by a fusion event. In this way, we have constructed a hypothetical ancestral karyotype, consisting of nine chromosomes, using the top 24 CARs identified in this investigation 


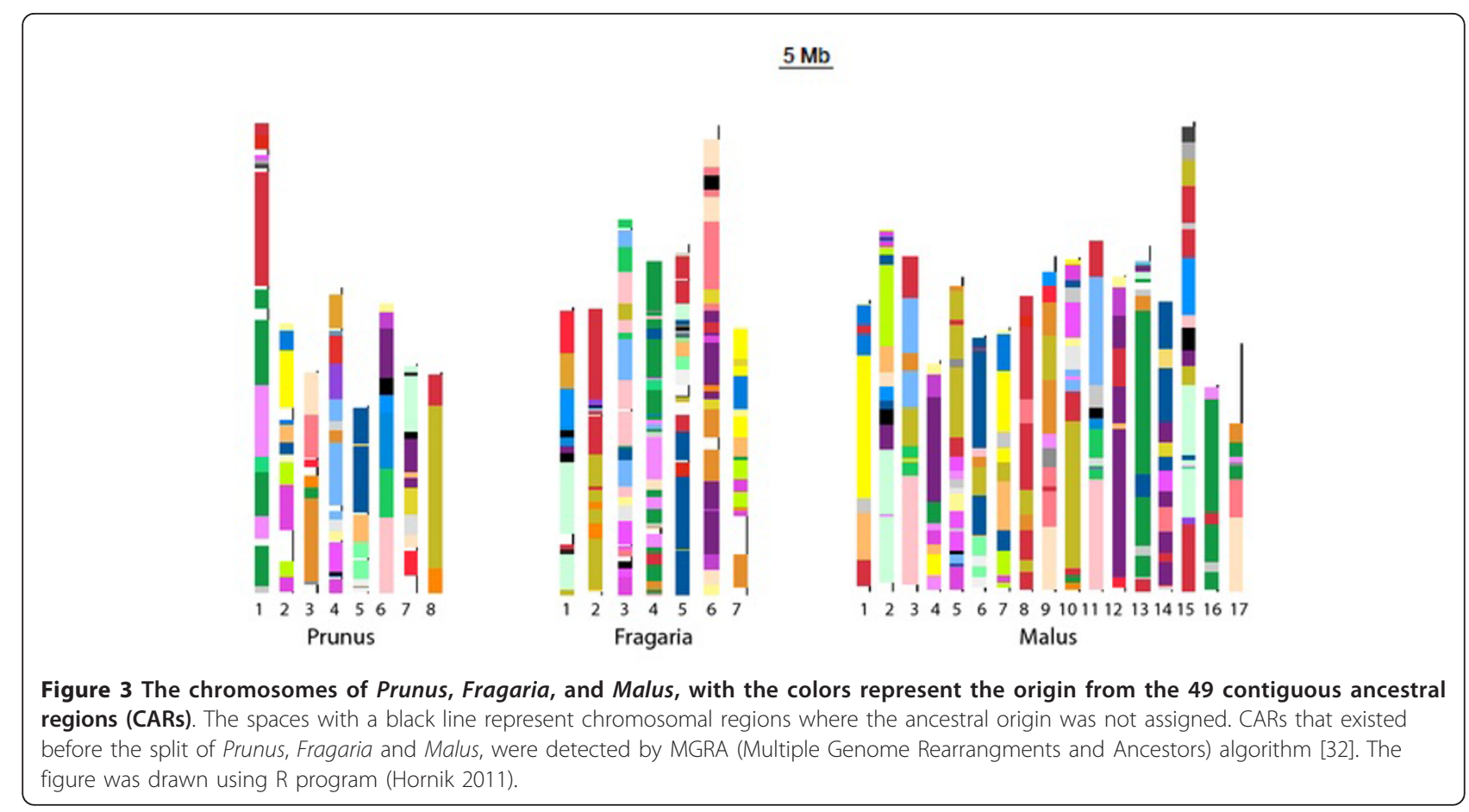

(Figure 4). The orthology maps between the three species, which support the hypothesis, are shown in Additional file 2: Figure S2. Figure 4 shows that the Fragaria lineage went through at least five fission events and seven fusion events, not including intrachromosomal rearrangements, the Prunus lineage went through at least three

Table 4 Number of breaks between chromosomal regions that are originated from different CARs

\begin{tabular}{|c|c|c|c|c|c|}
\hline \multicolumn{2}{|l|}{ Malus } & \multicolumn{2}{|l|}{ Prunus } & \multicolumn{2}{|l|}{ Fragaria } \\
\hline chromosome & No. break & chromosome & No. break & chromosome & No. break \\
\hline 1 & 5 & scaffold_1 & 26 & LG1 & 14 \\
\hline 2 & 12 & scaffold_2 & 11 & LG2 & 9 \\
\hline 3 & 8 & scaffold_3 & 6 & LG3 & 12 \\
\hline 4 & 8 & scaffold_4 & 15 & LG4 & 37 \\
\hline 5 & 15 & scaffold_5 & 8 & LG5 & 25 \\
\hline 6 & 8 & scaffold_6 & 8 & LG6 & 15 \\
\hline 7 & 10 & scaffold_7 & 12 & LG7 & 15 \\
\hline 8 & 6 & scaffold_8 & 5 & & \\
\hline 9 & 9 & & & & \\
\hline 10 & 13 & & & & \\
\hline 11 & 9 & & & & \\
\hline 12 & 6 & & & & \\
\hline 13 & 13 & & & & \\
\hline 14 & 9 & & & & \\
\hline 15 & 14 & & & & \\
\hline 16 & 7 & & & & \\
\hline 17 & 6 & & & & \\
\hline Sum & 158.0 & & 91.0 & & 127.0 \\
\hline Avg. (per $10 \mathrm{Mbp}$ ) & 3.0 & & 4.2 & & 6.4 \\
\hline Avg. (per chromosome) & 9.3 & & 11.4 & & 18.1 \\
\hline
\end{tabular}




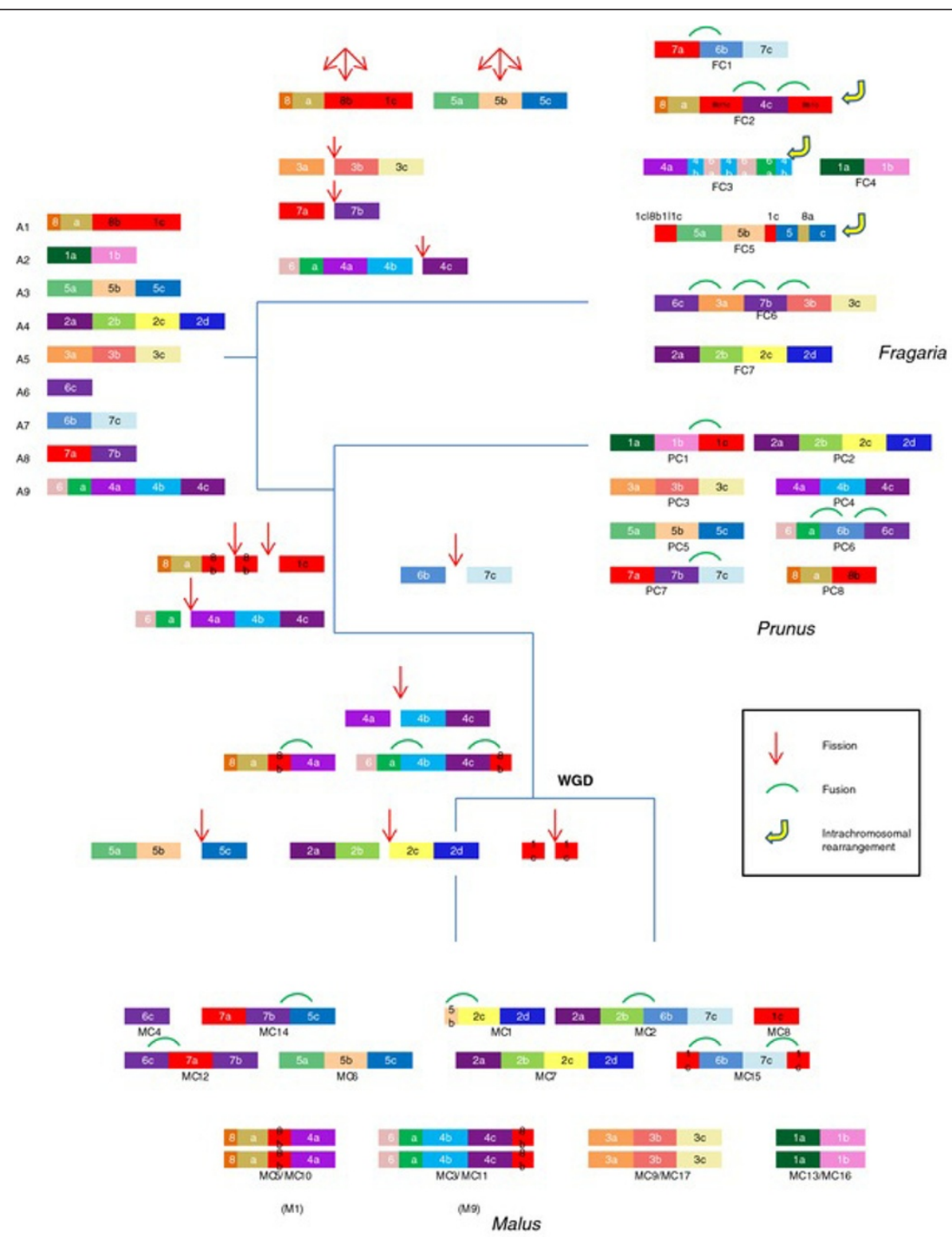

Figure 4 Hypothetical evolutionary steps from the nine Rosaceae ancestral chromosomes to Fragaria, Prunus and Malus lineage. Each color represent distinct CARs detected by MGRA algorithm. Chromosomal rearrangements specific for Rosoideae (contains Fragaria) and

Spireaoideae (contains Malus and Prunus) are depicted. Also shown are chromosomal rearragenments specific for Prunus, Malus, and subgenome of Malus after the WGD.

fission events and four fusion events and the Malus lineage went through seven fission events and nine fusion events. Two fission events occurred after the split of Fragaria and before the split of Malus and Prunus. Two further fission events and three fusion events occurred before the WGD of Malus lineage and the three further fission events occurred after the WGD in only one of the two homeologous chromosomes (Figure 4) of Malus. These data suggest that the Prunus lineage has the most conserved karyotype of the three species investigated and that the Malus lineage went through the most large-scale chromosomal fission/fusion events. It is also clear that intrachromosomal genome rearrangements played an important role in the genome evolution of the genus Fragaria. Additionally, Figure 4 suggests that the karyotypes of the ancestor of Malus existed before the WGD, as M1, M9 and A2 to A8. M1 and M9 were generated from A1 and $\mathrm{A} 9$, after four fissions and three fusions, and correspond to the present Malus chromosomes MC5/MC10 and $\mathrm{MC} 3 / \mathrm{MC} 11$, respectively. Our result is consistent with previous phylogenetic analyses $[21,35]$ and the analysis of comparative mapping data [2], in suggesting that 
both the ancestors of Rosaceae and Malus have genomes consisting of nine chromosomes.

To show how the genomes of the three taxa have evolved since they diverged from this common ancestral karyotypes, the nine ancestral chromosomes, A1 through A9, along with genomes of three species, colored by the ancestral chromosomal origin, were constructed (Additional file 4: Figure S3). In this figure, the 24 CARs in Figure 4 were reassigned with colors based on which of the nine ancestral chromosomes they reside in. The orthologous relationships amongst the three Rosaceae genomes are shown in the Rosaceae concentric circle with the putative nine chromosomes of Rosaceae ancestral genome as the innermost circle (Figure 5). This allows the identification of orthologous regions between the three genomes that have a common ancestral origin.

\section{Conclusions}

The availability of whole genome sequence data has permitted for the first time a detailed evaluation of the conservation of macro- and micro-synteny in the Rosaceae which has demonstrated that the genomes of Fragaria, Malus and Prunus have undergone different modes of evolution since they diverged from a common ancestor. This study has revealed that a greater number of small scale rearrangements have occurred in Fragaria than in either Malus or Prunus and has indicated that Malus went through more translocations potentially as a consequence of the WGD event in the lineage of the genus. The results of this investigation suggest that Prunus has the most conserved karyotype at both the macro- and micro-syntenic level in relation to the ancestral genome configuration for the Rosaceae, which in concordance with other studies is hypothesised to have had nine chromosomes. The resolution obtained in this comparison of genome structure demonstrates the utility of whole genome sequencing data to the elucidation of mechanisms driving genome evolution between related organisms at a level of resolution that would not have been possible through conventional comparative mapping endeavours.

\section{Materials and methods}

\section{Detection of orthologous regions}

To detect orthologous regions between the peach and grape genomes, the whole genome sequence and annotation data of grape were downloaded from Genoscope [36]. Whole genome sequence of Prunus persica v1.0, primary assembly of Malus domestica and Fragaria vesca beta version FvH4 pseudochromosomes were downloaded from GDR, Genome Database for Rosaceae [37,38]. The annotation data that includes the prediction of exons and genes were also downloaded from the databases above. All the sequence and annotation files that have been used in this study are available from GDR http://www.rosaceae.org/ BMC_rosaceae_Genome_paper. The whole genome sequences of peach and grape were masked for repeats using RepeatMasker [39], as well as the nmerge, WUBLAST distribution, and faSoftMask distribution utilities of Mercator [25]. Mercator identifies orthologous regions with one to one ortholgy relationships, rather than producing any syntenic regions in which one region can have many syntenic regions. Mercator employs BLAT-similar anchor pairs to identify orthologous segments in a modified k-way reciprocal best hit algorithm [40]. Translated sequences of exons, provided by the annotation data, have been used as anchors in these analyses. Two exons from each genome were determined to be similar if the BLAT [41] score of the pair was below 1e -10. BLAT scores were computed in protein space. To select the optimal criteria to assess conservation of synteny between Rosaceous genomes, Mercator parameters were varied from between a minimum of 30 exons and a maximum distance of 300 kbp between exons, to a minimum of two exons and a maximum distance of $3 \mathrm{Mbp}$ between exons. As the parameters become less stringent, we observed a sudden increase of the number of orthologous regions without the accompanying increase of the percent geonome coverage. Parameters selected for further analysis were a minimum of ten exons and a maximum distance of $300 \mathrm{kbp}$ between exons as these parameters gave high percentage coverage within the genomes but reduced small-size syntenic regions that are potentially artefactual. With the exception of the analysis shown in Figure 1, the Malus genome was split into two arbitrary 'sub-genomes' based on the data of Velasco et al [21]; sub-genome 1 consisted of chromosomes $1,2,3,4,5,8,9,13$ and 14, whilst sub-genome 2 was composed of chromosomes $6,7,10,11,12,15,16$ and 17 to use as an input for the Mercator program. This was done to detect orthologous regions in each of the homeologous Malus chromosomes. The anchored position of RosCOS markers in the peach genome were downloaded from GDR $[37,38]$. RosCOS markers were anchored to orthologous regions when their anchored positions in peach belong to the corresponding positions of ORs.

\section{Reconstruction of hypothetical ancestral genome}

We used the Multiple Genome Rearrangements and Ancestors (MGRA) algorithm [32] to predict Contiguous Ancestral Regions (CARs) that existed in a common ancestor. The orthology map of Prunus, Fragaria and Vitis genomes, produced by Mercator, was used as an input for the MGRA program. The Vitis genome was included in the analysis as anoutgroup. The hypothetical ancestral genome was manually constructed using CARs generated from MGRA, as written in the Result and discussion section above. 


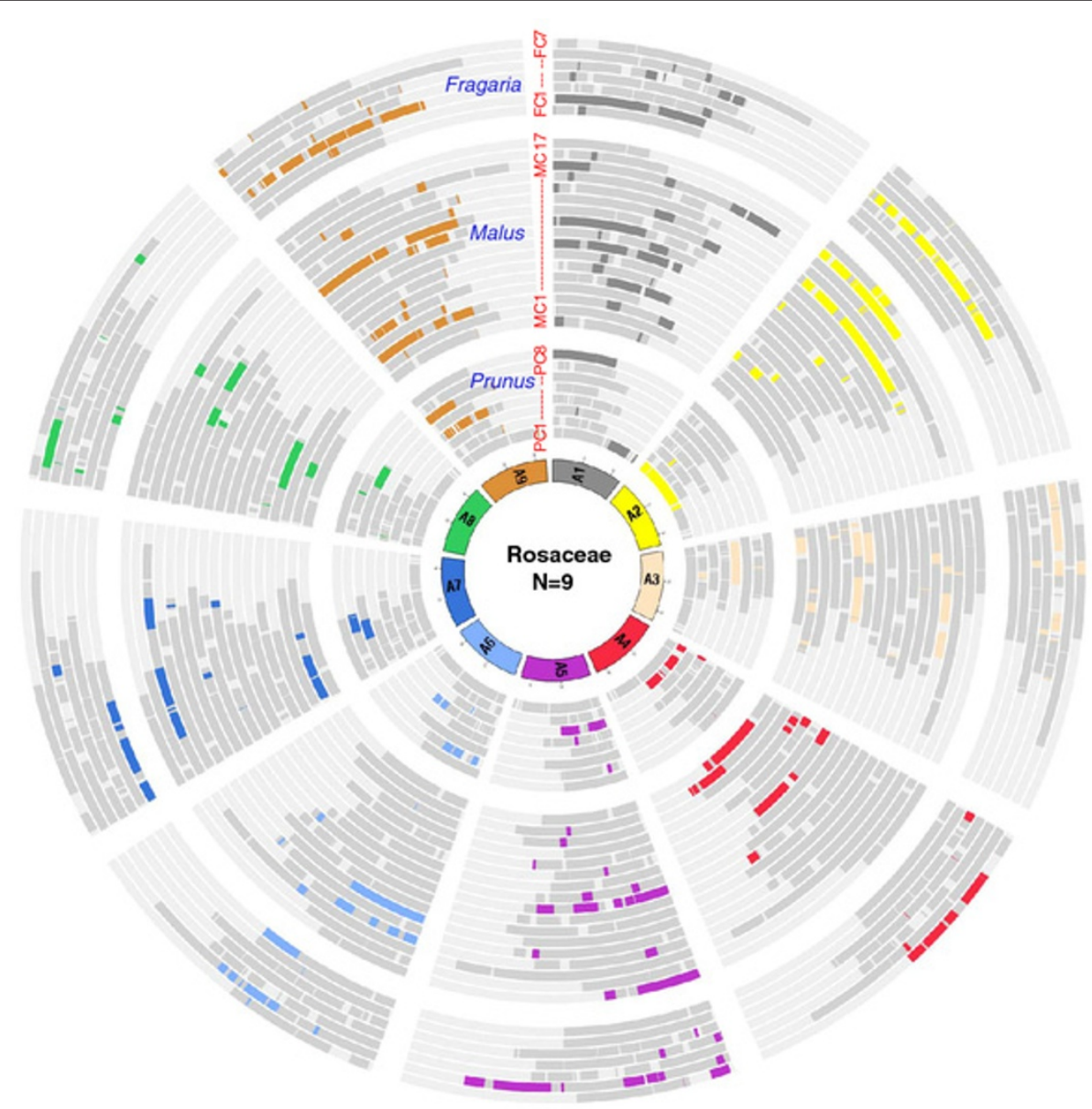

Figure 5 The Concentric circle of Rosaceae genomes. The innermost circle represents the putative nine chromosomes of Rosaceae ancestral genome. Next sets of circles represent eight, 17 and seven chromosomes of Prunus, Malus and Fragaria, respectively. The regions originated from each Rosaceae ancestral chromosome are highlighted with corresponding color in Figure S3. The Data were plotted using Circos [42].

\section{Additional material}

\section{Additional file 3: Table S1: List of ORs that are conserved in all} three genomes with their positions and orientations in each game

Additional file 1: Figure S1. Comparison of orthologous regions (OR) from two-species analysis and those from the three-species analysis. ORs between a Prunus chromosome (A:PC1, B:PC3, C:PC4, D:PC5, E:PC6, F:PC7, G:PC8) and chromosomes of Fragaria and Malus, detected from two separate analyses are shown in the diagram on the left. The same ORs shown in the diagram on the left as well as ORs that are shared by all three species are shown in the diagram on the right. Blue lines link the ORs shared by all three species, red lines link ORs between Prunus and Fragaria only, and green lines link ORs between Prunus and Malus only. Data with PC2 is shown in Figure 2 of the main manuscript. Data were plotted using Circos (Krzywinski et al. 2009).

Additional file 2: Figure S2. Orthology map identified between Prunus and the other two Rosaceous genera based on whole genome sequence analysis. The lines link one to one orthologous region identified using Mercator program (Dewey 2007). Only the orthologous regions between the major orthologous chromosomes, as shown in Table 2, are depicted. The colors represent the contiguous ancestral regions (CARs). The spaces with a black line represent chromosomal regions where the ancestral origin was not assigned. CARs that existed before the split of Prunus, Fragaria and Malus, were detected by MGRA (Multiple Genome Rearrangments and Ancestors) algorithm (Alekseyev and Pevzner 2009).
A through $\mathrm{H}$ shows orthologous regions in Fragaria and Malus corresponding to those in Prunus chromosome 1 through 8, respectively.

Additional file 4: Figure S3. The chromosomes of Prunus, Fragaria, and Malus, with the colors represent the origin from the nine putative chromosomes of Rosaceae ancestor. The spaces with a black line represent chromosomal regions where the ancestral origin was not assigned. For this figure, the top 24 CARs in Figure 4 were assigned to a distinct color, depending on which of the nine chromosomes of Rosaceae ancestor they belong to. The figure was drawn using $\mathrm{R}$ program (Hornik 2011).

\section{Abbreviations}

CARs: Contiguous ancestral regions; MGRA: Multiple genome rearrangements and ancestors; OR: Orthologous region; PID: Percentage identity; RosCOS: Rosaceous conserved orthologous sequences; SSR: Simple sequence repeat; WGD: Whole genome duplication.

\section{Acknowledgements}

We thank Colin Dewey (University of Wisconsin-Madison), Max Alekseyev (University of South Carolina), and Martin Krzywinski (Genome Sciences Center) for their advice on using programs, Mercator, MGRA and Circos, respectively. This project has been supported by the USDA NIFA SCRI grant 
\# 2010-2010-03255. We acknowledge International Peach Genome Initiative for the permission to use the peach genome in this study.

\section{Author details}

'Department of Horticulture and Landscape Architecture, Washington State University, Pullman, WA 99164, USA. ${ }^{2}$ Istituto Agrario San Michele all'Adige, Via E. Mach 1, 38010 San Michele all'Adige, Italy. ${ }^{3}$ Computer Science, Saginaw Valley State University, University Center, MI 48710, USA. ${ }^{4}$ Horticultural Sciences Department, University of Florida, Gainesville, Florida 32611, USA. ${ }^{5}$ Department of Horticultural Science, North Carolina State University, Campus Box 7609, Raleigh, NC 27695, USA. ${ }^{6}$ Department of Genetics and Biochemistry, Clemson University, Clemson, SC 29634, USA. 7 UMR Génétique et Horticulture (GenHort), INRA/Agrocampus-ouest/ Université d'Angers, Centre Angers-Nantes, 42 rue Georges Morel -, BP 60057, 49071 Beaucouzé cedex, France. ${ }^{8}$ IRTA, Centre de Recerca en Agrigenòmica CSIC-IRTA-UAB-UB, Campus UAB, Bellaterra (Cerdanyola del Vallès), 08193 Barcelona, Spain. ${ }^{\circ}$ Department of Biological Sciences, University of North Texas, 1155 Union Circle, Denton, Texas, USA. ${ }^{10} \mathrm{CRA}$ Fruit Tree Research Center, Via di Fioranello, 52, 00134 Rome, Italy. ${ }^{11}$ Istituto di Genomica Applicata, Parco Scientifico e Tecnologico L. Danieli, via Linussio, 51, 33100 Udine, Italy. ${ }^{12}$ DOE Joint Genomics Institute, 2800 Mitchell Dr, Walnut Creek, CA, USA.

\section{Authors' contributions}

SJ designed the study, performed the analysis, analyzed the data and wrote the paper. AC and MT participated in the design of the study, analyzed the data and critically revised the manuscript. DM participated in the design of the study and critically revised manuscript. PZ made figures that show contiguous ancestral regions using $\mathrm{R}$ program. IC wrote scripts for parsing data from Mercator output. KF, BS, AA, JMC, PA, VS, MM, DR, IV and RV conceived of the study and critically revised the manuscript. DS participated in the design of the study, analyzed the data and participated in writing. All authors read and approved the final manuscript.

\section{Competing interests}

The authors declare that they have no competing interests.

Received: 21 September 2011 Accepted: 4 April 2012 Published: 4 April 2012

\section{References}

1. Potter D, Gao F, Bortiri PE, Oh SH, Baggett S: Phylogenetic relationships in Rosaceae inferred from chloroplast matK and trnL-trnF nucleotide sequence data. Plant Syst Evol 2002, 231:77-89.

2. Vilanova S, Sargent DJ, Arus P, Monfort A: Synteny conservation between two distantly-related Rosaceae genomes: Prunus (the stone fruits) and Fragaria (the strawberry). BMC Plant Biol 2008, 8:67.

3. Illa E, Sargent DJ, Lopez Girona E, Bushakra J, Cestaro A, Crowhurst R, Pindo M, Cabrera A, van der Knaap E, lezzoni $A$, et al: Comparative analysis of rosaceous genomes and the reconstruction of a putative ancestral genome for the family. BMC Evol Biol 2011, 11:9.

4. Salse J, Bolot S, Throude M, Jouffe V, Piegu B, Quraishi UM, Calcagno T, Cooke R, Delseny M, Feuillet C: Identification and characterization of shared duplications between rice and wheat provide new insight into grass genome evolution. Plant Cell 2008, 20:11-24.

5. Salse J, Abrouk M, Bolot S, Guilhot N, Courcelle E, Faraut T, Waugh R, Close TJ, Messing J, Feuillet C: Reconstruction of monocotelydoneous proto-chromosomes reveals faster evolution in plants than in animals. Proc Natl Acad Sci USA 2009, 106:14908-14913.

6. The International Brachypodium Initiative: Genome sequencing and analysis of the model grass Brachypodium distachyon. Nature 2010, 463:763-768.

7. Jaillon O, Aury JM, Noel B, Policriti A, Clepet C, Casagrande A, Choisne N, Aubourg $S$, Vitulo $N$, Jubin $C$, et al: The grapevine genome sequence suggests ancestral hexaploidization in major angiosperm phyla. Nature 2007, 449:463-467.

8. Kaul S, Koo HL, Jenkins J, Rizzo M, Rooney T, Tallon LJ, Feldblyum T, Nierman W, Benito MI, Lin XY, et al: Analysis of the genome sequence of the flowering plant Arabidopsis thaliana. Nature 2000, 408:796-815.

9. Tuskan GA, Difazio S, Jansson S, Bohlmann J, Grigoriev I, Hellsten U, Putnam N, Ralph S, Rombauts S, Salamov A, et al: The genome of black cottonwood, Populus trichocarpa (Torr. \& Gray). Science 2006, 313:1596-1604.

10. Kalo P, Seres A, Taylor SA, Jakab J, Kevei Z, Kereszt A, Endre G, Ellis THN, Kiss GB: Comparative mapping between Medicago sativa and Pisum sativum. Mol Genet Genomics 2004, 272:235-246.

11. Lukens L, Zou F, Lydiate D, Parkin I, Osborn T: Comparison of a Brassica oleracea genetic map with the genome of Arabidopsis thaliana. Genetics 2003, 164:359-372.

12. Devos KM, Gale MD: Genome relationships: The grass model in current research. Plant Cell 2000, 12:637-646.

13. Doganlar S, Frary A, Daunay MC, Lester RN, Tanksley SD: A comparative genetic linkage map of eggplant (Solanum melongena) and its implications for genome evolution in the Solanaceae. Genetics 2002, 161:1697-1711.

14. Wu FN, Eannetta NT, Xu YM, Plieske J, Ganal M, Pozzi C, Bakaher N, Tanksley SD: COSII genetic maps of two diploid Nicotiana species provide a detailed picture of synteny with tomato and insights into chromosome evolution in tetraploid N. tabacum. Theor App/ Genet 2010, 120:809-827

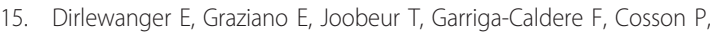
Howad W, Arús P: Comparative mapping and marker-assisted selection in Rosaceae fruit crops. Proc Natl Acad Sci USA 2004, 101:9891-9896.

16. Joobeur T, Viruel MA, de Vicente MC, Jauregui B, Ballester J, Dettori MT, Verde I, Truco MJ, Messeguer R, Batlle I, et al: Construction of a saturated linkage map for Prunus using an almond x peach F2 progeny. Theor Appl Genet 1998, 97:1034-1041.

17. Maliepaard C, Alston FH, van Arkel G, Brown LM, Chevreau E, Dunemann F, Evans KM, Gardiner S, Guilford P, van Heusden AW, et al: Aligning male and female linkage maps of apple (Malus pumila Mill.) using multi-allelic markers. Theor Appl Genet 1998, 97:60-73.

18. Sargent DJ, Rys A, Nier S, Simpson DW, Tobutt KR: The development and mapping of functional markers in Fragaria and their transferability and potential for mapping in other genera. Theor Appl Genet 2007, 114:373-384.

19. Sargent DJ, Marchese A, Simpson DW, Howad W, Fernandez-Fernandez F, Monfort A, Arus P, Evans KM, Tobutt KR: Development of "universal" genespecific markers from Malus spp. cDNA sequences, their mapping and use in synteny studies within Rosaceae. Tree Genet Genomes 2009, 5:133-145

20. Celton JM, Chagne D, Tustin SD, Terakami S, Nishitani C, Yamamoto T, Gardiner SE: Update on comparative genome mapping between Malus and Pyrus. BMC Res Notes 2009, 2:182.

21. Velasco R, Zharkikh A, Affourtit J, Dhingra A, Cestaro A, Kalyanaraman A, Fontana P, Bhatnagar SK, Troggio M, Pruss D, et al: The genome of the domesticated apple (Malus x domestica Borkh.). Nat Genet 2010, 42:833-839.

22. Cabrera A, Kozik A, Howad W, Arus P, lezzoni AF, van der Knaap E: Development and bin mapping of a Rosaceae Conserved Ortholog Set (COS) of markers. BMC Genomics 2009, 10:562.

23. Shulaev V, Korban SS, Sosinski B, Abbott AG, Aldwinckle HS, Folta KM, lezzoni A, Main D, Arús P, Dandekar AM, et al: Multiple models for Rosaceae genomics. Plant Physiol 2008, 147:985-1003.

24. Sosinski B, Jung S, Verde I, Schmutz J, Scholl E, Staton M, Abbott AG, Main D, Morgante M, Rokhsar D: The peach genome sequence and its utility for comparative genomics. Plant \& Animal Genomes XVIII Conference, January 9-13, 2010. San Diego, CA.

25. Dewey CN: Aligning multiple whole genomes with Mercator and MAVID. Methods Mol Biol 2007, 395:221-236.

26. Schmidt R: Plant genome evolution: lessons from comparative genomics at the DNA level. Plant Mol Biol 2002, 48:21-37.

27. Bennetzen JL: Patterns in grass genome evolution. Curr Opin Plant Biol 2007, 10:176-181

28. Buggs RJ, Doust AN, Tate JA, Koh J, Soltis K, Feltus FA, Paterson AH, Soltis PS, Soltis DE: Gene loss and silencing in Tragopogon miscellus (Asteraceae): comparison of natural and synthetic allotetraploids. Heredity 2009, 103:73-81.

29. Edger PP, Pires JC: Gene and genome duplications: the impact of dosage-sensitivity on the fate of nuclear genes. Chromosome Res 2009, 17:699-717. 
30. Kassahn KS, Dang VT, Wilkins SJ, Perkins AC, Ragan MA: Evolution of gene function and regulatory control after whole-genome duplication: comparative analyses in vertebrates. Genome Res 2009, 19:1404-1418.

31. Jiao Y, Wickett NJ, Ayyampalayam S, Chanderbali AS, Landherr L, Ralph PE, Tomsho LP, Hu Y, Liang H, Soltis PS, et al: Ancestral polyploidy in seed plants and angiosperms. Nature 2011, 473:97-100.

32. Alekseyev MA, Pevzner PA: Breakpoint graphs and ancestral genome reconstructions. Genome Res 2009, 19:943-957.

33. Sargent DJ, Fernandéz-Fernandéz F, Ruiz-Roja JJ, Sutherland BG, Passey A, Whitehouse $A B$, Simpson DW: A genetic linkage map of the cultivated strawberry (Fragaria $x$ ananassa) and its comparison to the diploid Fragaria reference map. Molecular Breeding 2009, 24:293-303.

34. Spigler RB, Lewers KS, Johnson AL, Ashman TL: Comparative mapping reveals autosomal origin of sex chromosome in octoploid Fragaria virginiana. J Heredity 2010, 101:S107-S117.

35. Evans RC, Campbell CS: The origin of the apple subfamily (Maloideae; Rosaceae) is clarified by DNA sequence data from duplicated GBSSI genes. Am J Bot 2002, 89:1478-1484.

36. Genoscope. [http://www.genoscope.cns.fr]]

37. Jung S, Staton M, Lee T, Blenda A, Svancara R, Abbott A, Main D: GDR (Genome Database for Rosaceae): integrated web-database for Rosaceae genomics and genetics data. Nucleic Acids Res 2008, Database: 1034-1040.

38. Genome Database for Rosaceae. [http://www.rosaceae.org/]

39. Smit AF, Hubley R, Green P: RepeatMasker Open-3.0. 1996 [http://www. repeatmasker.org].

40. Hirsh $A E$, Fraser $H B$ : Protein dispensability and rate of evolution. Nature 2001, 411:1046-1049.

41. Kent WJ: BLAT-the BLAST-like alignment tool. Genome Res 2002, 12:656-664.

42. Krzywinski M, Schein J, Birol I, Connors J, Gascoyne R, Horsman D, Jones SJ, Marra MA: Circos: an information aesthetic for comparative genomics. Genome Res 2009, 19:1639-1645.

doi:10.1186/1471-2164-13-129

Cite this article as: Jung et al:: Whole genome comparisons of Fragaria, Prunus and Malus reveal different modes of evolution between

Rosaceous subfamilies. BMC Genomics 2012 13:129.

\section{Submit your next manuscript to BioMed Central and take full advantage of:}

- Convenient online submission

- Thorough peer review

- No space constraints or color figure charges

- Immediate publication on acceptance

- Inclusion in PubMed, CAS, Scopus and Google Scholar

- Research which is freely available for redistribution

Submit your manuscript at www.biomedcentral.com/submit 